\title{
Effectiveness of Curcumin Therapy against Inflammation and Cancers
}

\section{Sajida Parveen Malik}

Department of Biomedical Engineering and Sciences, School of Mechanical and Manufacturing Engineering (SMME), National University of Sciences and Technology (NUST), H-12 Islamabad, Pakistan

*Corresponding author: Sajida Parveen Malik, Department of Biomedical Engineering and Sciences, School of Mechanical and Manufacturing Engineering (SMME), National University of Sciences and Technology (NUST), H-12 Islamabad, Pakistan, Tel: +92-313-5405198; E-mail: sajidamalik7@gmail.com

Received date: September 25, 2017; Accepted date: October 23, 2017; Published date: October 30, 2017

Copyright: (C) 2017 Malik SP. This is an open-access article distributed under the terms of the Creative Commons Attribution License, which permits unrestricted use, distribution and reproduction in any medium, provided the original author and source are credited.

\begin{abstract}
Plant isolated compounds have been in use to treat various ailments and to support normal physiological functions since long. Curcumin is also known for its beneficial health effects in the world of phytochemicals. Chemical name of curcumin is diferuloylmethane and it is extracted from turmeric and used for the prevention and cure of different inflammatory diseases. Inflammation is one of the root causes of diseases like arthritis, obesity, metabolic diseases like type II diabetes, pancreatitis, and neurodegenerative diseases cardiovascular even certain types of cancer. In Ayurveda medicine, turmeric has long been used for the treatment of different inflammatory disorders. Recent studies have shown that turmeric has an ingredient curcumin that is effectively applicable to therapeutic processes. Further, evidence has shown that inflammatory cell invasion, proliferation and angiogenesis can be inhibited using curcumin, through multiple molecular targets. Anti-inflammatory activity of Curcumin is carried out by suppressing of protein kinases, inflammatory cytokines, transcription factors, and enzymes that all promote inflammation, thus acts as a safe and non-toxic drug. However, curcumin's efficacy is hindered by poor absorption and low bioavailability, which limit its translation into clinics. This study summarizes anti-inflammatory and anticancer effects of curcumin.
\end{abstract}

Keywords Curcumin; Inflammation; Cancer; Inflammatory cytokines; Phytochemical

\section{Introduction}

In recent past, regardless of the hard work of doctors and scientists in decreasing the prevalence of fatal diseases like diseases of nervous system, circulatory system and digestive tract diseases, cancer and infectious diseases cannot be controlled at appreciable level [1]. In past few years, various drugs that are based on single target have been introduced, but these diseases are not an outcome of single reason or factor, rather multiple signalling pathways are known to be the cause of such above mentioned diseases. So, it is not effective to target only a single pathway, as such drugs are also not affordable in terms of price as well as in their side effects [2]. Thus, instead of drugs that are used to attack a single target, it is better to go for harmless, cost-effective, and freely available multi-target food components which provides protection from diseases as well as they are effective compounds for treatment of such diseases. About two centuries ago, Aggarwal et al. [3] and Shehzad et al. [4] discovered a dietary component Curcumin from turmeric (Curcuma longa). Curcumin (derived from turmeric) is a lipophilic agent which is stable in the acidic $\mathrm{pH}$ of stomach but quite insoluble in water. In few studies pure curcumin has been used but in most of the studies commercial turmeric or a mixture of curcuminoids have been used [5].

In traditional Indian medicine turmeric has been used to treat anorexia, hepatic disorders, biliary disorders, sinusitis, wound healing, inflammation and rheumatism $[6,7]$.

It has various beneficial roles including antibacterial potential first exhibited in 1949 [8]. Benefits of wound-healing, antioxidant and antiinflammatory activity, hypoglycemic activity anti-angiogenic and various apoptotic activities [9]. Curcumin undergoes sulphation and glucuronidation when administered orally and leads to the formation of hexahydrocurcumin, tetrahydrocurcumin and hexahydrocurcumino, when administered intravenously or via intraperitoneal routes. This difference in physiological and physiochemical properties exists due to the methoxy substitution on its aromatic ring [9].

Research has shown that curcumin can act together with molecules of many signaling pathways including cyclooxygenase-2 (COX-2) which is responsible for inflammation. And can mediate the modulation of growth factors along with their receptors, enzymes and various cytokines. These results prompt the clinical trials of its therapeutic effects.

After the discovery of these potential therapeutic effects of Curcumin, it has become a part of research in field of human health with keen interest of scientists. In a study, 5\% sodium Curcumin solution causes early filtration of gall bladder in human biliary disease. In addition to this, 67 patients of cholecystitis were given curcumin for 3 weeks with a complete cure for one of the patient with minimal side effects.

These trials also present its efficacy against broad range of diseases of humans including various cancers and inflammatory diseases. It was found very thoughtful for the scientists and doctors that how is it possible that an agent can solely possess numerous therapeutic and nutritious effects. Since, there is lot of study which gives proof of its vital roles in many signaling pathways [10].

Curcumin interacts with cell signaling molecules like proinflammatory cytokines, e.g. IL-1beta, TNF-alpha, IL-6, apoptotic proteins, (COX)-2, NF- $\kappa \mathrm{B}$, IKK $\beta$, STAT3, malondialdehyde (MDA), endothelin-1, E2, (CRP), PSA, GST, GSH, VCAM1, phosphorylase kinase $(\mathrm{PhK})$, pepsinogen, total cholesterol, transferrin receptor, 
triglyceride, transforming growth factor (TGF)- $\beta, \mathrm{HO}-1$, creatinine, ALT, AST and antioxidants [10].

Though studies on Curcumin are sometimes done on solely Curcumin, while rest are done by using mixtures or sometimes whole turmeric and afterwards. FDA has accepted Curcumin as safe and it is also being used as additional nutrient in many countries. Curcumin is used as capsules, tablets, energy beverages, and in makeup as well $[11,12]$.

The present review focuses on the in-vitro and in-vivo clinical trials of curcumin which mediates multiple molecular pathways. It describes possible health problems and potential complications related to curcumin treatment in case of diseases including diabetes, obesity, cardiovascular and Neurodegenerative diseases, cerebral edema, allergy, arthritis, inflammatory bowel disease, renal ischemia, scleroderma, psoriasis and various cancers.

\section{Molecular Mechanisms Associated with Use of Curcumin}

Various findings have demonstrated that curcumin binds to different molecular targets and affects cell signaling pathways. Curcumin is highly anti-inflammatory compound and directly interacts with cyclooxygenase 1 and 2 (COX-1,2) Tumor necrosis factor-alpha (TNF-a), reductase (ALR), thioredoxin reductase (TrxR) and antiapoptotic proteins including $\mathrm{Bcl} 2$ and $\mathrm{Bcl}-\mathrm{xL}$. It has also been shown to inhibit cytokines like Interleukins $1,2,6,8,12$, TNF-a, MAPK and JNK and can bind directly with DNA, RNA, proteins (immunoglobulin, albumins, caseins and divalent metal ions such as Manganese, Copper and Zinc, etc. [13].

Various transcription factors binds indirectly to Curcumin including activator protein-1 (AP-1) signal transducer and activator of transcription (STAT) and b-catenin proteins [14]. It has been shown to prevent nuclear factor-kappa B (NF-kB) recruitment, MMPs secretions, expressions of carboxylases and apoptosis inhibiting proteins like caspase- 3 and Bcl-2 family proteins. As it inhibits IL-1beta, NF-kB and PI3K/Akt are automatically suppressed by reducing phosphorylation and I-kB-alpha $[15,16]$.

These all targets of curcumin are either involved in prolonged inflammation or in the progression of different diseases including various respiratory tract diseases, i.e., asthma, skin diseases, i.e., psoriasis, neurodegenerative disorders or in the uncontrolled cell division in the case of various cancers. So, curcumin targeting various transcription factors and proteins are actually affecting the progression of such diseases and thus can be used in their treatment.

\section{Curcumin against Various Health Disorders}

Curcumin therapeutic effects includes its efficacy against numerous diseases like asthma, scleroderma, Inflammatory Bowel Disease, psoriasis, rheumatoid arthritis, neurodegenerative diseases, renal ischemia, cancers of many types and AIDS. These roles of Curcumin against above mentioned diseases are discussed in further detail as follows.

\section{Cancers}

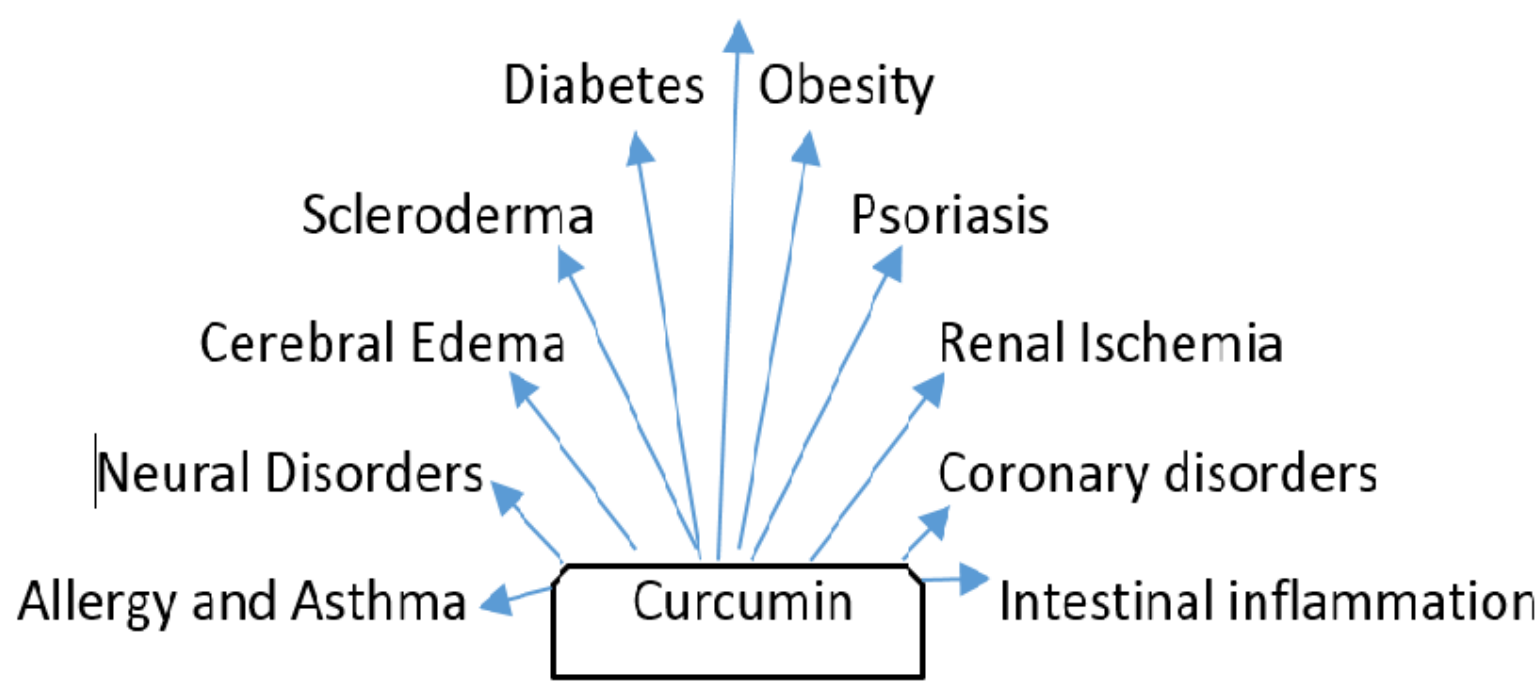

Figure 1: Curcumin therapy against diseases.

\section{Cancers}

Cancer is a multifaceted disease in which the initiation and progression is derived by an evolutionary process with a gradual accumulation of mutations at genetic and epigenetic level and are caused by dis-regulation of signaling pathways. This results into abnormal increase in cell proliferation without normal cell cycle regulation. Curcumin anti-cancer activity is associated with its targeted therapeutic effects on signaling molecules. Studies have shown that Curcumin possesses prospective therapeutic potential against many cancers including gastric cancer, colorectal cancer, breast cancer, hepatic cancer, intestinal cancer, pancreatic cancer, kidney cancer, bone cancer and brain cancer [17].

In all cancer types, inflammation is a major component of initiation and progression. Curcumin has been shown to be a potent anti- 
inflammatory agent with the inhibition of pro-inflammatory enzymes including COX-2 and inducible nitric oxide synthase (iNOS). Curcumin inhibits COX-2 activity by direct binding through Ala516, Val116, Val523 and Tyr355, COX-1 activity by direct binding through Ser15 while PGE2 suppression through hydrogen bonding with Ala562 [18].

In tumor progression, cell proliferation and transformation, cytokines, NF-jB and mitogen-activated protein kinases play an important role. Curcumin has been shown to suppress inflammatory cytokines including vascular endothelial growth factor (VEGF), HER2 oncoprotein, TNFa, and interleukin-1, 2, 6, 8, and 12 (IL-1, IL-2, IL-6, IL-8 and IL-12) and the activation of NF-jB [19].

T-cell factor (TCF), b-catenin and Lymphoid enhancer factor (LEF) are well known targets for many anti-cancer agents which can be targeted using Curcumin by overexpressing TCF- 4 in cancer cell lines and by decreasing nuclear translocation of b-catenin [20]. Matrix metalloproteinases (MMP-2, 9 and 14) have also been suppressed through the use of Curcumin which are associated with tumor growth, invasion and metastasis [21]. Expression of CC motif ligand 2 (CCl2) is down-regulated by curcumin along with STAT-3 and NF-jB suppression.

Curcumin has been shown to induce apoptosis involving Bax/ Bcl-2 with generation of ROS (Reactive oxygen species) with an enhancement of TRAIL induced apoptosis. Induction of Poly (ADPribose) polymerase (PARP) cleavage and activation of caspase 7, 9 by down regulation of inflammatory markers, inhibition of $\mathrm{PKC}$, epidermal growth factor (EGF)-receptor tyrosine kinase, association of cyclin D1 with cyclin-dependent kinase 4/6 (CDK4/CDK6), down regulation of PAK1 and phosphorylation of CDK-mediated retinoblastoma protein $(\mathrm{pRb})$ have been shown to suppress cancer cell proliferation [22].

\section{Diabetes}

Diabetes is a metabolic disorder and a hyperglycemic condition which affects liver, heart, kidneys and even brain. Diabetes is initiated and progressed by recruitment and execution of different cytokines, transcription factors and enzymes [22]. As we know that underlying mechanism of diabetes progression is damage of pancreatic beta cells, and Curcumin possesses anti-inflammatory activity and is anti-oxidant as well, so it reduces glucose level in blood by raising anti-oxidant condition in pancreas as it triggers the release of peroxisome proliferator- activated receptor gamma [23] $0.5 \%$ of curcumin if used as a diet could ameliorate the fasting blood glucose, urine volume and urine sugar in STZ induced diabetic rats [24]. A study conducted on diabetes model mice presented that Curcumin has anti-diabetic activity and it enhances diabetic condition associated with obesity by suppression of macrophage recruitment and up-regulation of adiponectin expression [25].

A study conducted on diabetes type 2 mice presented that Curcumin can also decrease blood glucose level through improvement of ligand binding activity in PPAR-c [26]. Studies conducted on diabetic mice showed that Curcumin reduces cholesterol levels in serum and liver, free fatty acids, phospholipid, VLDL and LDL expressions leading to suppression of hyperlipidemia and by the regulation of HDL cholesterol [27].

Curcumin can prevent liver damage by oxidative stress by amplification of enzymes possessing anti-oxidant activity and by causing down-regulation of superoxide dismutase, glutathione peroxidase and NADPH [28]. It has been introduced as therapeutic agent in therapy of liver fibrosis caused as a result of type 2 diabetes. Its role in signaling pathways as anti-diabetic agent is accomplished as it causes down regulation of ERK and stimulates AGE-R1 by triggering PPARc [28].

Curcumin was also evaluated for its protection against hyperalgesia in diabetic mice. Results presented Curcumin regulated the expression of TNF-alpha and it was found effective in reducing thermal sensational pain [29].

In another study, Curcumin has lowered the levels of VEGF release at the transcription level as well as translational level in retina with diabetic condition [30].

Transforming growth factor-betal, p300 and NF-kB causes enhanced release of ECM proteins. These proteins are associated with diabetic nephropathy. Curcumin thus decreases ECM proteins production by suppressing expression of p300 and thus treats nephropathy. In addition to this, Curcumin was also found to treat diabetic nephropathy by another mechanism in which it down regulates NO synthase enzyme, reduces TGF-beta 1 and endothelin-1 expressions. Diabetic nephropathy is known to cause suppression of p38 protein and HSP-27 which is responsible for elevated levels of nitrogen and creatinine and decreased levels of albumin in blood. Curcumin therapy is effective in lowering the release of creatinine and nitrogen and improving albumin concentration in blood. Histone $\mathrm{H} 3$ expression at protein level is also inhibited by Curcumin [31]. Curcumin plays an important role in the attenuation of symptoms associated with diabetes like in diabetic encephalopathy, the cognitive ability of rats can be protected [31]. Diabetic patients have several complications like liver diseases, coronary and pancreatic dysfunction. These have also shown to be treated by curcumin. In a study it was found that STZ induced diabetic rats excreted less creatine, urea, albumin and inorganic phosphorus when fed with dietary curcumin for a period of 8 weeks. This treatment also reduced weight of the liver and products of lipid peroxidation in plasma and urine [32].

Curcumin have many beneficial roles regarding pancreatic cells. In a study conducted by Alwi et al. curcumin has delayed ROS production and increased viability of islet cells mediated through the inhibition of poly ADP-ribose polymerase- 1 activation in the case of STZ induced islet damage [33].

These stimulatory actions of curcumin along with others including normalizing pancreatic GLUT2 levels, glucose clearance and cytokine (TNF, IL-1 and interferon-)-induced NF-B translocation by inhibiting phosphorylation of kappa B (IB) could contribute towards hypoglycemia in diabetes [34-36].

\section{Obesity}

Obesity is caused by abnormal increased weight of the body leading to many other health issues and decreases overall quality of life. Cooccurring diseases like cancer, type 2 diabetes, liver abnormalities, cardiovascular disease and obesity are linked to inflammation [37] and several studies have proved curcumin as an anti-inflammatory agent. Studies have shown that curcumin has the ability to inhibit signaling pathways related to obesity like Wnt/b-catenin and MAPK which resulted into upregulatio of cyclin D1 and c-Myc mRNA levels [38].

Curcumin has shown an important role in decreasing angiogenesis and adipogenesis by lowering cholesterol level and supressesing 
CCAAT/enhancer binding protein alpha and PPARc expression [39]. Curcumin is also known to cause suppression of macrophage recruitment in adipose tissue, leptin and leptin receptor levels in obesity disorder when associated with inflammation. Studies have shown that adiponectin when negatively regulated might decreases obesity and curcumin has the ability to increase the expression of adiponectin, by suppressing NF-KB release and metabolic and inflammatory markers [40]. One of the studies conducted for evaluation of effects of Curcumin results showed that Curcumin can enhance forbearance of high glucose levels through negative regulation of oxidative stress [41].

\section{Heart Diseases}

One of the major inflammatory Cardiovascular Disease (CVDs) is atherosclerosis, in which walls of blood vessels damages due to oxidative stress, and lipids accumulates in arteries and cells. LDL cholesterol oxidation is also involved in the progression of atherosclerosis [41]. Studies have shown that most of the cardiovascular disorders are associated with inflammation [42].

As already mentioned, Curcumin is anti-inflammatory agent and thus can be used to treat inflammatory atherosclerosis by triggering heme-oxygenase-1 via stimulation of ARE and hinders differentiation of endothelial and smooth cells of vessels. In aortic and vascular smooth muscle cells, Curcumin plays anti-inflammatory role by upregulation of p21and down-regulation of TNF-alpha cells through heme-oxygenase-1 enzyme release [43]. A study conducted with saltsensitive Dahl rats as experimental models, Curcumin was found to protect failure of sensitive heart by enhancing systolic heart function In an experiment on rat myocardium, Curcumin have been used for the treatment of myocardial ischemia which interacts with JAK/STAT3 pathway, caspase and Bcl-2 pathways. This interaction decreases the number of apoptotic cardiomyocytes, lessen lactate dehydrogenase release into the coronary flow and myocardial infarct size thus assists in an overall improvement in post-ischemic cardiac function [44].

Curcumin studies also reveal that it can prevent damage in abdominal aortic a-neuronal-systems in experiments, as it suppresses AP-1 and NF-kB binding ability to DNA and also impedes the release of interleukins like IL-6 and IL-1beta, and inhibits MMP-9 and MCP-1 in aortic tissues [44]. Curcumin regulates signaling processes to protect from inflammation, hypertrophy of cardiac tissues, and fibro genesis by inhibition of cytokines and p300 [45,46].

\section{Neural Diseases}

Neurodegenerative diseases can be elicited by abnormally regulated noxious proteins and injured neurons. These diseases accompany abnormal development pattern of proteins that results in gene mutations such as presenilins 1 or 2 or human amyloid precursor protein (hAPP) in Alzheimer's disease (AD), characterized by oxidative damage and inflammation [47]. In cultured hippocampal neurons, Curcumin have shown suppression of B-amyloid oligomers which are characteristic of disease progression with induction of tau phosphorylation through signaling of c-Jun $\mathrm{N}$ terminal kinase (JNK) [48].

Specifically, in the substantia nigra, curcumin was shown to protect tyrosine hydroxylase-positive cells and in the striatum it has maintained dopamine levels, possibly through its efficient dispersion into the brain and antioxidant activity. In another study, seizures prevention, oxidative stress, seizure-induced cognitive impairment and memory impairment and was achieved when curcumin was administered orally to pentylenetetrazole (PTZ)-induced kindled epileptic rat [48]. The development and differentiation of Th17 cells, responsible for initiating multiple sclerosis through the downregulation of IL-21, IL-6, STAT3 and NF-jB-phosphorylation, can also be inhibited through curcumin [49]. In the rat forebrain, curcumin has also shown to protect against ischemia-reperfusion injury by suppressing superoxide anion production, xanthine oxidase activity, GPx, SOD, malondialdehyde level and lactate dehydrogenase activities [50]. Haloperidol when chronically administered in rats, increases tongue protrusion, facial jerking and vacuous chewing movements by modulating their antioxidant system. However, curcumin pretreatment showed repressed effects which were dose dependent. The reduced levels of serotonin, norepinephrine and dopamine levels in the subcortical and cortical regions due to haloperidol chronic administration were improved (increased) when curcumin was administered [51]. Neurochemical analyses have also confirmed the role of curcumin in the protection of Creutzfeld-Jakob disease (spongiform encephalopathies) with the help of binding prion protein $(\operatorname{PrP})$. Recently, for treatment of neuropathic pain, curcumin antinociceptive effects were investigated. It was revealed that at least in part, the curcumin anti-nociceptive effects, is through the inhibition of monoamine system that is coupled with 5 -HT1A receptor and spinal b2-adrenoceptor [52].

\section{Cerebral Edema}

Poor prognosis of traumatic brain injuries causes osmotic differences and structural damage which are the main cause of cerebral edema. [53]. When brain edema was measured following blood-brain barrier disruption and intra-cerebral hemorrhage curcumin treatment decreased blood-brain barrier disruption, prevented intra-cerebral hemorrhage and brain edema through the modulation of MMP cells [54]. Moreover, the role of curcumin in inflammatory pathways and oxidative stress has been investigated in hypoxia-induced models of cerebral edema. These hypoxic conditions reduce the activity of antioxidant enzyme followed by increased proinflammatory cytokines, levels of cell adhesion molecules and gene expression of NF-кB. Moreover, the levels of brain NF-jB have been decreased by curcumin pre-treatment $(100 \mathrm{mg} / \mathrm{kg})$ and hypoxia-induced cerebral edema was markedly attenuated [55].

\section{Allergy and Bronchial Asthma}

Inflammatory cytokines mediates these pro-inflammatory diseases like allergy and asthma. It has been reported that repression of mast cell histamine through the use of curcumin can provide protection from different allergies [56]. In curcumin a functional group of hydroxyl in diferuloylmethane has shown decreased allergic reactions, increased antioxidant levels and improved constricted airways in allergy and asthma model [55]. In another study, curcumin was used to treat intragastrically $\mathrm{BALB} / \mathrm{c}$ mice (a latex allergy model) overexpressing Th2 type immune response. Curcumininhibited Th2 responses followed by peripheral blood eosinophilia, decreased expression of co-stimulatory molecules (CD86, OX40L and CD80) on MMP-9, antigen-presenting cells and suppression of lung inflammation and thymic stromal lymphopoietin and ornithine amino transferase [55]. Furthermore, in chronic obstructive pulmonary disease (COPD) and asthma, oxidative stress disruption results in the resistance to steroid therapy through the unbalanced acetylation and deacetylation of histone deacetylase and activation of NF-jB. 
Curcumin scavenges free radicals like $\mathrm{O} 2$ and NO, acting as a powerful antioxidant through decreased activation of NF-кB and MAPK and as well as down-regulates pro-inflammatory mediators like growth factor receptor genes, MMPs and adhesion molecules in inflammatory lung disease [57]. In non-typeable Hemophilus influenzae (NTHi) (induced by COPD), Curcumin was found to reduce tumor progession in lungs, increasing neutrophil chemoattractant keratinocyte in bronchoalveolar lavage fluid that are derived chemokines and neutrophils. Anti-tumor effects like colony formation, apoptosis and cell viability has been shown in an in vitro study using curcumin [58].

\section{Intestinal Inflammation}

It is a disorder of immune system with debilitating involvement of chronic inflammation of digestive tract, including Crohn's disease (CD) and ulcerative colitis (UC). In some chemically induced colitis in vitro and in vivo models, curcumin has been found to reduce colitis [59]. Curcumin not only prevents translocations of NF-jB but also inhibits 5-LOX, iNOS and COX-2 expression in IBD. In experimental colitis; it also suppresses TLR4-induced NF-jB activation [60].

The curcumin treatment results were investigated on five approved patients- with UC/proctitis and five patients with CD. The UC/ proctitis group has reduced erythrocyte sedimentation rate and $\mathrm{CD}$ activity index (CDAI) whereas in $\mathrm{CD}$ a protein, $\mathrm{C}$-reactive protein was observed. With the reduction of cramping, abdominal pain, diarrhea with improved bowel movements, curcumin treated group showed improvement [61,62]. Curcumin was shown to reduce diseaseassociated clinical activity index, decreased the relapse rate and the endoscopic index in UC [63]. All these evidences shows curcumin efficacy for the prevention and treatment of Inflammatory Bowel Diseases.

\section{Renal Ischemia}

Renal ischemia also known as reperfusion injury (IRI) is an interconnected and complex result of renal transplantation, mainly caused by poor prognosis and as a result acute kidney injuries. This disease is characterized by increase in inflammatory markers in kidney as a result of NF-кB activation and oxidative stress [64]. In skin allograft models, curcumin played an important role separately or in concomitant administration. Curcumin was shown to increase serum creatinine levels, prolongs skin graft survival and a decrease in renal inflammation and tubular damage. Curcumin has shown to induce apoptosis through down-regulation of TNF-a, heat shock protein-70 and TLR-4 expression with normal levels of serum creatinine and improved injury healing. Curcumin also reduced superoxide generation and enhanced SOD through the suppression of phospho-S6 ribosomal protein, NF-jB and MAPK. It also inhibited inflammatory chemokine activation and neutrophil infiltration in the renal IRI [65].

\section{Scleroderma}

An autoimmune rheumatic disease that typically results in skin fibrosis, vasculopathy and fibrosis of other organs, with an abnormal regulation of NF-кB and inflammatory cytokines involved in fibrosis and angiogenesis. In scleroderma lung fibroblasts (SLF) regulated by epsilon isoform of protein kinase C (PKCe), treatment with curcumin induces apoptosis through inducting GST P1 and HO-1. Curcumin selectively regulates phase 2 detoxification enzymes in normal fibroblasts, SLFs and in fibrotic lung tissue (in vivo), mediating expression of PKCe [66]. Curcumin also selectively inhibits the TGF- $\beta$ - induced Smad2 phosphorylation and in patient cells of systemic scleroderma, it induced apoptosis through up-regulation of TGF- $\beta$ induced factor (TGIF). Curcumin can decrease ubiquitination of TGIF as TGIF is a negative regulator of TGF- $\beta$ signaling, thus inhibiting TGF $\beta$ altogether [67].

\section{Psoriasis}

Psoriasis is a chronic inflammatory disease causing inflammation of skin by abnormal proliferation and differentiation of keratinocytes and have various inflammatory markers including surviving, NF-jB, TNF- $\alpha$ and STAT3 [68]. Studies have also suggested that curcumin may provide protection against psoriasis in psoriatic keratinocytes, by decreasing the pro-inflammatory cytokines expression and inhibiting the keratinocyte proliferation. Recently, it has been shown that in TRAIL and TNF- $\alpha$ stimulated HaCaT cells, curcumin treatment induces apoptosis through the anti-apoptotic proteins down-regulation such as Bcl-xL and inhibitor of apoptosis protein 1 and 2 (IAP1, IAP2) with inhibition of NF-jB subunit p65 activation. These results show that curcumin can be used to treat psoriasis [69].

\section{Curcumin Clinical Study}

In different cancers the efficacy, safety and pharmacokinetics of curcumin have been addressed in different in vitro and in vivo studies regarding chemoprevention and other therapeutic effects [70]. When patients with various inflammatory diseases were treated with curcumin, it has been reported that it reduces or even eliminates the growth of different diseases including cancer. The most effective curcumin results were observed in patients with post-operative inflammation, chronic anterior uveitis, orbital pseudo tumors, pancreatic cancer and idiopathic inflammatory external cancer lesion. Recent clinical trials confirmed the curcumin anticancer activity in human subjects including pre-neoplastic diseases, neoplastic such as pancreatic cancer, multiple myeloma, and colon cancer and myelodysplastic syndromes [71]. Curcumin anti-inflammatory activity has also been observed in various inflammatory disorders including hyperglycemia, arthritis, dermatitis, cystic fibrosis, cardiovascular and liver diseases, several brain diseases like schizophrenia, Alzheimer and various pathological conditions of orthodontics and periodontics.

\section{Curcumin Limitations}

Recently the development of curcumin as a nutraceutical for inflammatory diseases treatment has gained significant attention [72]. After oral administration its decreased bioavailability supports the study of its half-life and extremely low serum levels. During several in vitro studies involving curcumin, the concentrations of the drug usually ranges in micro-molar range while the plasma concentrations of curcumin involving high oral doses are relatively very low, mainly in nano-molar range. This counts for main hindrance for the clinical development of oral curcumin. The low clinical efficiency in case of diseases including Cardiovascular and Alzheimer's disease has been shown in a study [73]. While for cancers the poor prolonged delivery of curcumin at high doses and reduced bioavailability accounts for viable cancer cells even in treated conditions [74]. As a result, several methods have been considered to improve the retention and bioavailability of curcumin. For enhancing its bioavailability the most common strategy is the use of adjuvants that can block curcumin metabolic pathway. Various studies have shown that curcumin's phospholipid complexes its preparation as nanoparticles (curcumin 
structural analogs and nanocurcumin) also enhance systemic bioavailability [74]. However methods to improve levels of curcumin are also linked with the increased toxicity under specific conditions [75]. Goodpasture and Arrighi [75] reported a dose and timedependent induction of chromosome aberrations in various mammalian cell lines at concentrations of $10 \mathrm{ug} / \mathrm{ml}$. Since then various studies have confirmed the DNA damage associated with more or less similar concentration of curcumin raising concern about its safety. These effects may involve several possible mechanisms including Reactive oxygen species (ROS), i.e., hydrogen peroxide and superoxide anion which plays an important role in the progression of cancer and other diseases as explained earlier [76]. This is due to the presence of 2 a-b-unsaturated ketones in the structure of curcumin which reacts with exposed thiol groups of cysteine residues of proteins explaining the production of ROS and as a result DNA damage. Curcumin could also produce undesired increase of drugs in plasma as it has been shown to inhibit the drug metabolizing enzymes (cytochrome P450, UDP-glucuronosyltransferase and glutathione-S-transferase) in a study. Other safety issues involve the role of curcumin as active iron chelator in vivo causing iron deficiency anaemia in mice 58 making it in- effective in people with iron suboptimal levels.

Therefore for now the number of studies showing positive effects of curcumin is much higher than the number of negative effects of curcumin study which does not necessarily means that the benefit ratio of curcumin is higher than the risk ratio but it may also indicates that there is more work going on for the evaluation of beneficial aspects of curcumin than its toxicity. So, in order to develop curcumin as an effective drug its benefit-risk ratio should be fully researched and acknowledged.

\section{Conclusion}

Curcumin is a potential anti-inflammatory phytochemical that can be used to treat inflammatory disorders and various cancers. Its effective dose is very important to be known prior to application of curcumin therapy against a particular disorder. Because its overuse have been demonstrated to be disadvantageous. However, it has been one of those phytochemicals that are frequently being used against various inflammatory diseases since years. Further, its use should be extended for treatment and prevention of cancers.

\section{References}

1. Shehzad A, Khan S, Sup Lee Y (2012) Curcumin molecular targets in obesity and obesity-related cancers. Future Oncol 8: 179-190.

2. Frantz S (2005) Drug discovery: Playing dirty. Nature 437: 942-943.

3. Aggarwal BB, Kumar A, Bharti AC (2003) Anticancer potential of curcumin: Preclinical and clinical studies. Anticancer Res 23: 363-398.

4. Shehzad A, Iqbal W, Shehzad O, Lee YS (2012) Adiponectin: Regulation of its production and its role in human diseases. Hormones (Athens) 11: 8-20.

5. Singh S (2007) From exotic spice to modern drug? Cell 130: 765-768.

6. Singh R, Sharma P (2011) Hepatoprotective effect of curcumin on lindane-induced oxidative stress in male wistar rats. Toxicol Int18: 124.

7. Aggarwal BB, Sung B (2009) Pharmacological basis for the role of curcumin in chronic diseases: An age-old spice with modern targets. Pharmacol Sci 30: 85-94.

8. Schraufstatter E, Bernt H (1949) Antibacterial action of curcumin and related compounds. Nature 164: 456-457.
9. Somparn P, Phisalaphong C, Nakornchai S, Unchern S, Morales NP (2007) Comparative antioxidant activities of curcumin and its demethoxy and hydrogenated derivatives. Biol Pharm Bull 30: 74-78.

10. Goel A, Jhurani S, Aggarwal BB (2008) Multi-targeted therapy by curcumin: How spicy is it? Mol Nutr Food Res 52: 1010-1030.

11. Gupta SC, Patchva S, Koh W, Aggarwal BB (2012) Discovery of curcumin, a component of golden spice and its miraculous biological activities. Clin Exp Pharmacol Physiol 39: 283-299.

12. Chandran B, Goel A (2012) A randomized, pilot study to assess the efficacy and safety of curcumin in patients with active rheumatoid arthritis. Phyto Res 26: 1719-1725

13. Gupta SC, Prasad S, Kim JH, Patchva S, Webb LJ, et al. (2011) Multitargeting by curcumin as revealed by molecular interaction studies. Nat Prod Rep 28: 1937-1955.

14. Shehzad A, Lee YS (2010) Curcumin: Multiple molecular targets mediate multiple pharmacological actions: A review. Drugs Fut 35: 113.

15. Buhrmann C, Mobasheri A, Busch F, Aldinger C, Stahlmann R et al. (2011) Curcumin modulates nuclear factor kappaB (NF-kappaB)mediated inflammation in human tenocytes in vitro: Role of the phosphatidylinositol 3-kinase/Akt pathway. J Biol Chem 286: 28556-28566.

16. Goel A, Aggarwal BB (2010) Curcumin, the golden spice from Indian saffron, is a chemosensitizer and radiosensitizer for tumors and chemoprotector and radioprotector for normal organs. Nutr Cancer 62: 919-930.

17. Padhye S, Banerjee S, Chavan D, Pandye S, Swamy KV, et al. (2009) Fluorocurcumins as cyclooxygenase-2 inhibitor: molecular docking, pharmacokinetics and tissue distribution in mice. Pharm Res 26: 2438-2445.

18. Aggarwal BB, Vijayalekshmi R, Sung B (2009) Targeting inflammatory pathways for prevention and therapy of cancer: short-term friend, longterm foe. Clin Cancer Res 15: 425-430.

19. Park CH, Hahm ER, Park S, Kim HK, Yang CH (2005) The inhibitory mechanism of curcumin and its derivative against ß-catenin/Tcf signaling. FEBS letters 579: 2965-2971.

20. Shehzad A, Wahid F, Lee YS (2010) Curcumin in cancer chemoprevention: molecular targets, pharmacokinetics, bioavailability and clinical trials. Arch Pharm 343: 489-499.

21. Sa G, Das T (2008) Anti-cancer effects of curcumin: Cycle of life and death. Cell Div 3: 14.

22. Kowluru RA, Chan PS (2007) Oxidative stress and diabetic retinopathy. Exp Diabetes Res 2007: 43603.

23. Kajimoto Y, Kaneto H (2004) Role of oxidative stress in pancreatic betacell dysfunction. Ann N Y Acad Sci 1011: 168-176.

24. Chougala MB, Bhaskar JJ, Rajan MGR, Salimath PV (2012) Effect of curcumin and quercetin on lysosomal enzyme activities in streptozotocin-induced diabetic rats. Clin Nutri 31: 749-755.

25. Weisberg SP, Leibel R, Tortoriello DV (2008) Dietary curcumin significantly improves obesity-associated inflammation and diabetes in mouse models of diabesity. Endocrinology 149: 3549-3558.

26. Nishiyama T, Mae T, Kishida H, Tsukagawa M, Mimaki Y, et al. (2005). Curcuminoids and sesquiterpenoids in turmeric (Curcuma longa L.) suppress an increase in blood glucose level in type 2 diabetic KK-Ay mice. J Agri Food Chem 53: 959-963.

27. Pari L, Murugan P (2007) Anti-hyperlipidemic effect of curcumin and tetrahydrocurcumin in experimental type 2 diabetic rats. Ren Fail 29: 881-889.

28. Lin J, Tang Y, Kang Q, Feng Y, Chen A (2012) Curcumin inhibits gene expression of receptor for advanced glycation end-products (RAGE) in hepatic stellate cells in vitro by elevating PPAR? activity and attenuating oxidative stress. Br J Pharmacol 166: 2212-2227.

29. Sharma S, Kulkarni SK, Agrewala JN, Chopra K (2006) Curcumin attenuates thermal hyperalgesia in a diabetic mouse model of neuropathic pain. Eur J Pharmacol 536: 256-261. 
30. Mrudula T, Suryanarayana P, Srinivas PN, Reddy GB (2007) Effect of curcumin on hyperglycemia-induced vascular endothelial growth factor expression in streptozotocin-induced diabetic rat retina. Biochem Biophys Res Commun 361: 528-532.

31. Tikoo K, Meena RL, Kabra DG, Gaikwad AB (2008) Change in posttranslational modifications of histone $\mathrm{H} 3$, heat-shock protein-27 and MAP kinase p38 expression by curcumin in streptozotocin-induced type I diabetic nephropathy. Br J Pharmacol 153: 1225-1231.

32. Babu PS, Srinivasan K (1995) Influence of dietary curcumin and cholesterol on the progression of experimentally induced diabetes in albino rat. Mol Cell Biochem 152: 13-21.

33. Alwi I, Santoso T, Suyono S, Sutrisna B, Suyatna FD, et al. (2008) The effect of curcumin on lipid level in patients with acute coronary syndrome. Acta Med Indones 40: 201-210.

34. Kanitkar M, Gokhale K, Galande S, Bhonde RR (2008) Novel role of curcumin in the prevention of cytokine-induced islet death in vitro and diabetogenesis in vivo. Br J Pharmacol 155: 702-713.

35. Shehzad A, Ha T, Subhan F, Lee YS (2011) New mechanisms and the antiinflammatory role of curcumin in obesity and obesity-related metabolic diseases. Eur J Nutr 50: 151-161.

36. Premanand C, Rema M, Sameer MZ, Sujatha M, Balasubramanyam M (2006) Effect of curcumin on proliferation of human retinal endothelia cells under in vitro conditions. Invest Ophthalmol Vis Sci 47: 2179-2184.

37. Meghana K, Sanjeev G, Ramesh B (2007) Curcumin prevents streptozotocin-induced islet damage by scavenging free radicals: A prophylactic and protective role. Eur J Pharmacol 577: 183-191.

38. Ahn J, Lee H, Kim S, Ha T (2010) Curcumin-induced suppression of adipogenic differentiation is accompanied by activation of Wnt/ß-catenin signaling. Am J Physiol Cell Physiol 298: 1510-1516.

39. Ejaz A, Wu D, Kwan P, Meydani M (2009) Curcumin inhibits adipogenesis in 3T3-L1 adipocytes and angiogenesis and obesity in C57/BL mice. J Nutr 139: 919-925.

40. He HJ, Wang GY, Gao Y, Ling WH, Yu ZW, et al. (2012) Curcumin attenuates Nrf2 signaling defect, oxidative stress in muscle and glucose intolerance in high fat diet-fed mice. World J Diabetes 3: 94-104.

41. Rosner MH, Ronco C, Okusa MD (2012) The role of inflammation in the cardio-renal syndrome: A focus on cytokines and inflammatory mediators. Semin Nephrol 32: 70-78.

42. Yuan Y, Li P, Ye J (2012) Lipid homeostasis and the formation of macrophage-derived foam cells in atherosclerosis. Protein Cell 3: 173-181.

43. Wongcharoen W, Phrommintikul A (2009) The protective role of curcumin in cardiovascular diseases. Int J Cardiol 133: 145-151.

44. Morimoto T, Sunagawa Y, Kawamura T, Takaya T, Wada H, et al. (2008) The dietary compound curcumin inhibits p300 histone acetyltransferase activity and prevents heart failure in rats. J Clin Invest 118: 868-878.

45. Duan W, Yang Y, Yan J, Yu S, Liu J, et al. (2012) The effects of curcumin post-treatment against myocardial ischemia and reperfusion by activation of the JAK2/STAT3 signaling pathway. Basic Res Cardiol 107: 1-12.

46. Kim KH, Lee EN, Park JK, Lee JR, Kim JH, et al. (2012) Curcumin attenuates TNF- $\alpha$-induced expression of intercellular adhesion molecule-1, vascular cell adhesion molecule-1 and proinflammatory cytokines in human endometriotic stromal cells. Phytother Res 26:1037-47.

47. Smith DG, Cappai R, Barnham KJ (2007) The redox chemistry of the Alzheimer's disease amyloid beta peptide. Biochim Biophys Acta 1768: 1976-1990.

48. Mehla J, Reeta KH, Gupta P, Gupta YK (2010) Protective effect of curcumin against seizures and cognitive impairment in a pentylenetetrazole-kindled epileptic rat model. Life Sci 87: 596-603.

49. Xie L, Li XK, Takahara S (2011) Curcumin has bright prospects for the treatment of multiple sclerosis. Int Immunopharmacol 11: 323-330.

50. Ghoneim AI, Abdel-Naim AB, Khalifa AE, El-Denshary ES (2002) Protective effects of curcumin against ischaemia/reperfusion insult in rat forebrain. Pharmacol Res 46: 273-279.
51. Bishnoi M, Chopra K, Kulkarni SK (2008) Protective effect of Curcumin, the active principle of turmeric (Curcuma longa) in haloperidol-induced orofacial dyskinesia and associated behavioural, biochemical and neurochemical changes in rat brain. Pharmacol Biochem Behav 88: 511-522.

52. Zhao X, Xu Y, Zhao Q, Chen CR, Liu AM, et al. (2012). Curcumin exerts antinociceptive effects in a mouse model of neuropathic pain: descending monoamine system and opioid receptors are differentially involved. Neuropharmacology 62: 843-854.

53. Laird MD, Sukumari-Ramesh S, Swift AE, Meiler SE, Vender JR, et al. (2010) Curcumin attenuates cerebral edema following traumatic brain injury in mice: A possible role for aquaporin-4. J Neurochem 113: 637-648.

54. Sun Y, Dai M, Wang Y, Wang W, Sun Q, et al. (2011) Neuroprotection and sensorimotor functional improvement by curcumin after intracerebral hemorrhage in mice. J Neurotrauma 28: 2513-2521.

55. Himadri P, Kumari SS, Chitharanjan M, Dhananjay S (2010) Role of oxidative stress and inflammation in hypoxia-induced cerebral edema: A molecular approach. High Alt Med Biol 11: 231-244.

56. Kurup VP, Barrios CS (2008) Immunomodulatory effects of curcumin in allergy. Mol Nutr Food Res 52: 1031-1039.

57. Kurup VP, Barrios CS, Raju R, Johnson BD, Levy MB, et al. (2007) Immune response modulation by curcumin in a latex allergy model. Clin Mol Allergy 5: 1.

58. Biswas S, Rahman I (2008) Modulation of steroid activity in chronic inflammation: A novel anti-inflammatory role for curcumin. Mol Nutr Food Res 52: 987-994.

59. Moghaddam SJ, Barta P, Mirabolfathinejad SG, Ammar-Aouchiche Z, Garza NT, et al. (2009) Curcumin inhibits COPD-like airway inflammation and lung cancer progression in mice. Carcinogenesis 30: 1949-1956.

60. Belcaro G, Cesarone MR, Dugall M, Pellegrini L, Ledda A, et al. (2010). Efficacy and safety of Meriva ${ }^{\oplus}$, a curcumin-phosphatidylcholine complex, during extended administration in osteoarthritis patients. Altern Med Rev 15: 337-44.

61. Ali T, Shakir F, Morton J (2012) Curcumin and inflammatory bowel disease: biological mechanisms and clinical implication. Digestion 85: 249-255.

62. Holt PR, Katz S, Kirshoff R (2005) Curcumin therapy in inflammatory bowel disease: A pilot study. Dig Dis Sci 50: 2191-2193.

63. Ledda A, Belcaro G, Dugall M, Luzzi R, Scoccianti M, et al. (2012) Meriva $^{\oplus}$, a lecithinized curcumin delivery system, in the control of benign prostatic hyperplasia: A pilot, product evaluation registry study. Panminerva Med 54: 17.

64. Hanai H, Iida T, Takeuchi K, Watanabe F, Maruyama Y, et al. (2006) Curcumin maintenance therapy for ulcerative colitis: randomized, multicenter, double-blind, placebo-controlled trial. Clin Gastroenterol Hepatol 4: 1502-1506.

65. Patschan D, Patschan S, Müller GA (2012) Inflammation and microvasculopathy in renal ischemia reperfusion injury. J Transplant 2012: 764154 .

66. Jones EA, Shoskes DA (2000) The effect of mycophenolate mofetil and polyphenolic bioflavonoids on renal ischemia reperfusion injury and repair. J Urology 163: 999-1004.

67. Tourkina E, Gooz P, Oates JC, Ludwicka-Bradley A, Silver RM, et al. (2004) Curcumin-induced apoptosis in scleroderma lung fibroblasts: Role of protein kinase Ce. Am J Respir Cell Mol Biol 31: 28-35.

68. Song K, Peng S, Sun Z, Li H, Yang R (2011) Curcumin suppresses TGF- $B$ signaling by inhibition of TGIF degradation in scleroderma fibroblasts. Biochem Biophys Res Commun 411: 821-825.

69. Abdou AG, Hanout HM (2008) Evaluation of survivin and NF- $\kappa B$ in psoriasis, an immunohistochemical study. J Cutan Pathol 35: 445-451.

70. Sun J, Han J, Zhao Y, Zhu Q, Hu J (2012) Curcumin induces apoptosis in tumor necrosis factor-alpha-treated HaCaT cells. Int Immunopharmacol 13: $170-174$. 
Citation: Malik (2018) Effectiveness of Curcumin Therapy against Inflammation and Cancers. Biol Syst Open Access 7: 183. doi: 10.4172/2329-6577.1000183

Page 8 of 8

71. Shehzad A, Lee J, Lee YS (2013) Curcumin in various cancers. Biofactors 39: 56-68.

72. Syng-Ai C, Kumari AL, Khar A (2004) Effect of curcumin on normal and tumor cells: role of glutathione and bcl-2. Mol Cancer Ther 3: 1101-1108.

73. Anand P, Kunnumakkara AB, Newman RA, Aggarwal BB (2007) Bioavailability of curcumin: Problems and promises. Mol Pharm 4: 807-818.

74. Shoba G, Joy D, Joseph T, Majeed M, Rajendran R, et al. (1998) Influence of piperine on the pharmacokinetics of curcumin in animals and human volunteers. Planta Med 64: 353-356.
75. Sasaki H, Sunagawa Y, Takahashi K, Imaizumi A, Fukuda H, et al. (2011) Innovative preparation of curcumin for improved oral bioavailability. Biol Pharmaceutical Bull 34: 660-665.

76. Goodpasture CE, Arrighi FE (1976) Effects of food seasonings on the cell cycle and chromosome morphology of mammalian cells in vitro with special reference to turmeric. Food Cosmet toxicol 14: 9-14.

77. Cerutti PA (1985) Prooxidant states and tumor promotion. Science 227: $375-381$ 